\title{
Leading brand products and their supermarket economy line equivalents, is there a difference in nutritional content?
}

\author{
E. Jackson and S. Mushtaq \\ Department of Clinical Sciences and Nutrition, University of Chester, CHI 4BJ.
}

Since the introduction of supermarket economy lines (SELs) in the early 1990s, their popularity has been established nationwide ${ }^{(1)}$. However, these economical alternatives are commonly perceived to be of lower nutritional quality than their leading brand (LB) equivalents ${ }^{(2,3,4)}$. The present study aimed to determine if there is a significant difference in nutritional content between the UK top-selling LBs and their SEL equivalents. Additionally, the study aimed to investigate if on average, LBs or SELs provide better 'value for money'.

The LBs of 38 most popular food categories were identified from UK market research, and equivalent SEL products were identified from each of the retailers with the top-five majority UK market share: Tesco, Sainsbury's, Asda, Morrisons and Aldi. In each food category, differences between LBs and SELs in: energy, fat, saturated fatty acid, carbohydrate, sugar, fibre, protein and salt content, per $100 \mathrm{~g}$ of food product were determined using a one-sample T-test. The nutritional quality of each product was also determined by a nutrient profiling system. Cost was analysed in relation to shopping baskets containing 33 equivalent products. Six shopping baskets were analysed, one containing LB products and one from each SEL retailer. The cost of each shopping basket was calculated using pack price and price per $100 \mathrm{~g}$ or $100 \mathrm{~mL}$ of food product.

Data was collected for 219 products; 38 LBs and 181 SELs. 86 significant differences were identified in specific nutrients across the food categories, but the direction of the differences was inconsistent. Based on pack price, the total LB shopping basket cost was $£ 61.91$ whereas average SEL basket cost $£ 28.62$, a difference of $£ 33.29$ or $54 \%(\mathrm{P}=0.001)$. However, there was no difference between the nutrient profile of LBs and SELs.

Although significant differences were identified between nutrients in some food categories, overall, there appeared to be no difference in nutritional content between LBs and SEL equivalents. This association is consistent with previous studies and is contrary to the common perception that SELs are of lower nutritional quality than $\operatorname{LBs}^{(2,3,4,5,6)}$. Pertinent to public health, the present study found that SEL breakfast cereals contained a significantly higher amount of salt than the LB $(P=0.035)^{(4,6)}$. Additionally, although the majority of food categories did not show a significant difference in energy content per $100 \mathrm{~g}$ of food product (29 of 38 ) LB pasta had significantly higher energy content per $100 \mathrm{~g}$ of food product than SEL equivalents $(\mathrm{P}=0.017)^{(6)}$.

In conclusion, there appears to be no difference in nutritional content between the LB and SEL equivalents in 38 popular food categories, however, there appears to be twofold difference in price The cost analysis demonstrates that consumers can purchase the same quantity of foodstuff for significantly less when opting for SEL products. Low income households may therefore be encouraged to purchase SEL products to reduce weekly household expenditure and enable a greater proportion of the budget to be available for the purchase fresh produce such as fruit, vegetables and meat.

1. Collins-Dodd C, Lindley T (2003 Nov 1) Store brands and retail differentiation: the influence of store image and store brand attitude on store own brand perceptions. Journal of Retailing and Consumer Services 10(6), 345-52.

2. Ahuja JK, Pehrsson PR, Cogswell M (2017 May 1) A Comparison of Concentrations of Sodium and Related Nutrients (Potassium, Total Dietary Fiber, Total and Saturated Fat, and Total Sugar) in Private-Label and National Brands of Popular, Sodium-Contributing, Commercially Packaged Foods in the United States. J Acad Nutr Diet 117(5), 770-7.

3. Cooper S, Nelson M (2003 Oct 1) 'Economy' line foods from four supermarkets and brand name equivalents: a comparison of their nutrient contents and costs. JHND 16(5), 339-47.

4. Trevena H, Neal B, Dunford E et al. (2015 Aug 21) A comparison of the sodium content of supermarket private-label and branded foods in Australia. Nutrients 7(8), 7027-41.

5. Faulkner GP, Livingstone MB, McCaffrey TA, Kerr MA (2014 Dec 1) Supermarket own brand foods: Lower in energy cost but similar in nutritional quality to their market brand alternatives. $J H N D$ 27(6), 617-25.

6. Cleanthous X, Mackintosh AM, Anderson S (2011 Jun 1) Comparison of reported nutrients and serve size between private label products and branded products in Australian supermarkets. Nutr Diet 68(2), 120-6. 